\title{
Avaliação em pacientes portadores de diabetes mellitus tipo 2 atendidos pela Farmácia Municipal de Santa Maria, RS, Brasil: registros de intervenção farmacêutica
}

\author{
Evaluation in patients with type 2 diabetes mellitus attended by \\ the Municipal Pharmacy of Santa Maria, RS, Brazil: pharmaceutical \\ intervention records
}

Recebido em: 23/07/2021

Aceito em: $\quad 06 / 10 / 2021$
Gabriela Nunes FLORES; Luísa Pagliarini WEBER; Carine Viana SILVA; Paula Eliete Rodrigues BITENCOURT Departamento de Análises Clínicas e Toxicológicas, Centro de Ciências da Saúde, Universidade Federal de Santa Maria. Av. Roraima, $n^{\circ}$ 1000, prédio 26, Cidade Universitária, CEP 97105900. Santa Maria, RS, Brasil. E-mail: bitencourt.paula@yahoo.com.br

\section{ABSTRACT}

Type 2 diabetes mellitus (DM), characterized by hyperglycemia associated with defects in insulin secretion/action, affects a large number of individuals. Adequate glycemic control can reduce the risk of developing complications. However, as it is a silent disease, the time for diagnosis can facilitate the development of other diseases, which leads to the use of other drugs and, consequently, to drug interactions (MI). The objective of this study was to carry out a pharmaceutical intervention in the municipal pharmacy of Santa Maria, aiming at improving the glycemic control of patients with type $2 \mathrm{DM}$. Questionnaires were used to obtain data about the disease and the medications used by this population. Patients were followed up monthly (for three months). IM were searched in the Drugs database. The results show that most patients are women, over 50 years of age and the disease was diagnosed after 35 years of age. In addition, they have a family history of DM and already manifest some type of complication. The pharmacotherapeutic follow-up and health education services suggested a positive response to DM control in the treated patients. Regarding the most used medications, there were hypoglycemic agents, such as metformin and insulin, in addition to antihypertensives. A total of $323 \mathrm{MI}$ was found, 11 classified as severe. The results confirmed that pharmaceutical guidance greatly helps the patient to understand the influence of adherence to treatment and the inclusion of lifestyle changes in adequate glycemic control.

Keywords: type 2 diabetes; insulin dependents; municipal pharmacies; pharmaceutical intervention

\section{RESUMO}

Diabetes mellitus (DM) tipo 2, caracterizado por hiperglicemia associada a defeitos na secreção/ação da insulina, acomete um grande número de indivíduos. O controle glicêmico adequado pode reduzir o risco de desenvolvimento de complicações. Entretanto, por ser uma doença silenciosa, o tempo para o diagnóstico 
pode facilitar o desenvolvimento de outras doenças, o que leva a utilização de outros medicamentos e, consequentemente, a interações medicamentosas (IM). O objetivo desse trabalho foi realizar uma intervenção farmacêutica na farmácia municipal de Santa Maria, visando melhorar o controle glicêmico de pacientes com DM tipo 2. Foram utilizados questionários para obtenção de dados sobre a doença e os medicamentos utilizados por essa população. Os pacientes foram acompanhados mensalmente (por três meses). As IM foram pesquisadas na base de dados Drugs. Os resultados demonstraram que grande parte dos pacientes era mulher, acima de 50 anos e o diagnóstico da doença foi após os 35 anos. Além disso, apresentavam histórico familiar de DM e já manifestavam algum tipo de complicação. Os serviços de acompanhamento farmacoterapêutico e de educação em saúde realizados sugeriram uma resposta positiva ao controle do DM nos pacientes atendidos. Com relação aos medicamentos mais utilizados estavam os hipoglicemiantes, como a metformina e insulina, além de anti-hipertensivos. Foram encontradas 323 IM, sendo 11 classificadas como graves. Os resultados confirmaram que a orientação farmacêutica auxilia sobremaneira na compreensão pelo paciente sobre a influência da adesão ao tratamento e da inclusão de mudanças no estilo de vida no adequado controle glicêmico.

Palavras-chave: DM tipo 2; insulinodependentes; Farmácias municipais; intervenção farmacêutica.

\section{INTRODUÇÃO}

O diabetes mellitus (DM) tipo 2 é uma doença crônica não transmissível (DCNT) que acomete mais de 350 milhões de pessoas no mundo. Fatores de risco evitáveis, como a má alimentação e obesidade, contribuem para o número cada vez maior de pacientes acometidos por essa doença. O Brasil tem o maior número de pessoas com DM na América Latina, chegando a aproximadamente 16,8 milhões de casos $(1,2)$. Foram registradas 247.500 mil mortes por DM na América do Sul e mais da metade ocorreu no Brasil (1). De acordo com o Estudo Longitudinal de Saúde do Adulto (ELSA), cerca de 50\% dos pacientes diagnosticados com DM por meio de Teste de Tolerância Oral a Glicose (TOTG) não sabiam serem portadores da doença (3). Associado a esse fato, um estudo publicado por Eik Filho e cols (2016) demonstrou que a população brasileira possui grau de conhecimento insuficiente, além de ser alta a incidência de pacientes portadores de DM tipo 2 com controle glicêmico inadequado (4).

De acordo com a Sociedade Brasileira de Diabetes (SBD) (2020), após o diagnóstico de DM tipo 2, os pacientes são advertidos sobre mudanças em seus hábitos de vida e utilização de hipoglicemiantes orais (2). Entretanto, é evidente que existe perda gradual e progressiva do controle glicêmico, independente do tratamento instituído, sendo necessário o uso de insulina com a evolução da doença (2). Portanto, boa parte dos pacientes diabéticos tipo 2 precisarão fazer uso de insulina em algum momento.

A Lei Federal n 11.347/2006, regulamentada no Sistema Único de Saúde (SUS) pela Portaria $\mathrm{n}^{\circ} 2.583 / 2007$, assegura ao paciente diabético o direito ao fornecimento gratuito de medicamentos e materiais necessários para o monitoramento da glicemia capilar. Todavia, é condição para o recebimento dos medicamentos e materiais que o paciente esteja inscrito no programa de educação especial para diabéticos $(5,6)$. Por meio dessas ações, os pacientes são inseridos no processo terapêutico individual, incluindo acompanhamento clínico e seguimento farmacoterapêutico, formalizado por meio dos devidos registros em prontuário. A educação em saúde a estes pacientes é imprescindível para o controle adequado da doença e na melhora qualidade de vida.

Geralmente, as complicações associadas ao DM tipo 2 geram a necessidade de uso de uma variedade de fármacos, aumentando a ocorrência de interações medicamentosas (IM) que podem colocar em risco a vida do paciente $(7,8)$. Considerando o exposto juntamente o número alarmante de dados sobre a morbimortalidade dessa doença na população brasi- 
leira, é suma importância a atuação do farmacêutico, por meio de seus serviços especializados, no provimento de assistência capacitada para a promoção do autocuidado e o uso racional de medicamentos. Portanto, o objetivo desse trabalho foi realizar uma intervenção farmacêutica na farmácia municipal de Santa Maria, RS, por meio de um acompanhamento mensal de pacientes portadores de DM tipo 2, além da coleta de dados sociodemográficos e avaliação sobre as possíveis IM nessa população.

\section{MÉTODO}

A pesquisa se trata de um estudo transversal realizado com pacientes portadores de DM tipo 2, com diagnóstico previamente definido por médicos, atendidos pelo SUS na farmácia municipal central de Santa Maria, RS. A farmácia atendia cerca de 832 pacientes diabéticos cadastrados residentes nessa cidade. Cabe destacar que as farmácias municipais, sob responsabilidade técnica de um farmacêutico conforme a lei 5.991/1973, são responsáveis pela distribuição gratuita de medicamentos e insumos (como material para aplicação de insulina, glicosímetro portátil, fitas para a mensuração da glicemia e lancetas) aos pacientes, sendo necessário a apresentação de um documento de identificação e a prescrição médica.

Foram incluídos nesse estudo pacientes que frequentam regularmente a farmácia municipal, de ambos os gêneros, com idade entre 18 e 65 anos, portadores de DM tipo 2 que faziam uso de insulina. Os critérios de exclusão foram os seguintes: crianças, pacientes portadores de DM tipo $1 \mathrm{e}$ aqueles assistidos por cuidadores e/ou familiares.

Após assinatura do Termo de Consentimento Livre e Esclarecido (TCLE), durante a consulta farmacêutica realizada na própria farmácia, foi aplicado um questionário para adquirir informações sociodemográficas dessa população, bem como sobre a doença e tipos de medicamentos utilizados por essa população. A consulta farmacêutica está vinculada a projetos de pesquisa e extensão realizados pelo curso de farmácia da Universidade Federal de Santa Maria. Após essa etapa, os pacientes receberam uma cartilha contendo um diário, onde marcaram suas glicemias oriundas dos testes realizados diariamente por meio do glicosímetro portátil (Roche Accu-check active $\left.{ }^{\circledR}\right)$ distribuído pela farmácia e, também outras informações, como a prática de exercícios físicos. Os pacientes foram avaliados durante três meses (março a maio de 2019) através do preenchimento de questionários mensais e durante o retorno desses pacientes foi distribuído um novo diário. Após o primeiro mês, também foi realizada uma intervenção farmacêutica por meio do serviço de educação em saúde guiado por folders explicativos sobre prática de exercício físico e alimentação adequada, visando a adequação do controle glicêmico do usuário.

As IM entre os medicamentos utilizados por essa população foram avaliadas utilizando a base de dados Drugs (drugs.com, acesso online e gratuito), o qual as classifica quanto à gravidade em grave, moderada ou leve. A interação é classificada como leve quando resulta em um prejuízo clínico de menor significado. Já a moderada é aquela onde resultam em efeitos indesejáveis de importância clínica, sendo usado apenas em circunstâncias especiais. A IM considerada grave representa um agravo clínico altamente significativo, onde deve-se avaliar criteriosamente o risco benefício e se necessário manter os fármacos adotar medidas de suporte ao paciente.

Os resultados foram apresentados em frequência (\%) na forma de gráficos e tabelas com auxílio do software Microsoft Office Excel ${ }^{\circledR} 2019$. Os dados obtidos foram analisados e comparados com a literatura existente. Este trabalho foi aprovado pelo Comitê de Ética em Pesquisa da Universidade Federal de Santa Maria sob o número CAAE 99231218.4.0000.5346.

\section{RESULTADOS E DISCUSSÃO}

Foram analisados os dados de um total de 39 pacientes portadores de DM tipo 2 que utilizavam insulina e frequentavam mensalmente a farmácia municipal central de Santa Maria, RS. Na Tabela 1, estão os resultados sobre a caracterização sociodemográfica desses pacientes. Com a análise dessa tabela é possível observar que cerca de $64 \%$ dos pacientes eram do gênero feminino. Esse dado pode estar relacionado ao fato de que as mulheres têm um maior cuidado com a sua saúde, portanto, antecipando o diagnóstico da doença (9).

O DM tipo 2 tem maior prevalência em indivíduos com idade superior a 45 anos, pois as alterações biológicas do envelhecimento contribuem 


\section{lutarma}

para o desenvolvimento da doença - entre elas, o aumento da resistência à insulina - devido a redução da massa muscular e a redução da função pancreática $(2,10)$. Nosso estudo corrobora essa afirmação, uma vez que a maior parte da população estudada $(87,18 \%$ ) estava na faixa etária entre 51-70 anos. Com relação ao nível socioeconômico, mais da metade $(51,28 \%)$ realizaram somente o ensino fundamental e $71,79 \%$ vivia com renda de um salário mínimo ou menos. Ainda, utilizando a classificação do estado nutricional dos indivíduos conforme o IMC e o ponto de corte preconizado pela Organização Mundial de Saúde (OMS), foi observado que $61,53 \%$ dos pacientes encontravamse acima do peso, sendo $25,64 \%$ pré-obesos (IMC entre 25,0 a $\left.29,9 \mathrm{~kg} / \mathrm{m}^{2}\right), 28,20 \%$ com obesidade grau I (IMC entre 30,0 a $34,9 \mathrm{~kg} / \mathrm{m}^{2}$ ) e $7,69 \%$ com obesidade grau II (IMC entre 35,0 a $39,9 \mathrm{~kg} / \mathrm{m}^{2}$ ).

Entre os fatores de risco para o desenvolvimento do DM tipo 2 podemos citar o alto IMC, seguido de sedentarismo (11). Além disso, se sabe que uma dieta equilibrada e rica em produtos frescos é de grande importância para o equilíbrio e a manutenção da saúde de pacientes portadores de DM (2). Entretanto, o acesso a esses produtos pode ser muito dispendioso para grande parte da população, como demonstrado pela faixa salarial de grande parte dos pacientes aqui avaliados. Assim, o consumo de alimentos industrializados acaba sendo facilitado devido ao menor custo que esses apresentam e, também, ao tempo de preparo, o que pode estar relacionado com o significativo número de pacientes acima do peso encontrados em nosso estudo.

Em relação às comorbidades, é notável a presença de pacientes portadores de hipertensão $(89,74 \%)$, seja DM e hipertensão $(28,20 \%)$, hipertensão e dislipidemia (12,82\%) ou hipertensão e outras doenças $(48,72 \%)$. Esses resultados evidenciam as complicações geradas pelo DM, pois a hiperglicemia crônica está envolvida no desenvolvimento e progressão de muitas doenças, principalmente hipertensão e problemas renais, visto que afeta o sistema macro e microvascular (12).

Ainda com relação a Tabela 1 uma minoria $(10,26 \%)$ dos pacientes participantes desse estudo relatou o consumo de bebidas alcoólicas e cigarro, sendo esse fato de grande relevância nessa população que necessita de um controle estreito em seus hábitos de vida.
Tabela 1. Características sociodemográficas dos pacientes portadores de DM tipo 2 insulinodependentes que frequentavam a farmácia Municipal Central de Santa Maria, RS, Brasil, no período de março a maio de 2019.

\begin{tabular}{|c|c|c|}
\hline Caracteristicas & $\mathbf{N}$ & Frequência (\%) \\
\hline \multicolumn{3}{|c|}{ Gênero } \\
\hline Feminino & 25 & 64,1 \\
\hline Masculino & 14 & 35,9 \\
\hline \multicolumn{3}{|l|}{ Idade } \\
\hline 30 a 40 & 1 & 2,5 \\
\hline $40 a 50$ & 4 & 10,2 \\
\hline 50 a 60 & 15 & 38,4 \\
\hline 60 a 70 & 19 & 48,7 \\
\hline \multicolumn{3}{|c|}{ Escolaridade } \\
\hline Analfabeto & 2 & 5,1 \\
\hline Ensino fundamental & 20 & 51,2 \\
\hline Ensino médio & 14 & 35,9 \\
\hline Ensino superior & 3 & 7,6 \\
\hline \multicolumn{3}{|l|}{ Renda } \\
\hline Menos de 1 salário mínimo & 8 & 20,5 \\
\hline 1 & 20 & 51,2 \\
\hline 2 & 4 & 10,2 \\
\hline 3 & 4 & 10,2 \\
\hline Mais de 3 salários mínimos & 3 & 7,6 \\
\hline \multicolumn{3}{|c|}{ Fumante } \\
\hline Sim & 4 & 10,2 \\
\hline Não & 35 & 89,7 \\
\hline \multicolumn{3}{|c|}{ Consumo de álcool } \\
\hline Sim & 4 & 10,2 \\
\hline Não & 35 & 89,7 \\
\hline \multicolumn{3}{|c|}{ Doenças } \\
\hline Diabetes & 4 & 10,2 \\
\hline Diabetes e hipertensão & 11 & 28,2 \\
\hline Diabetes, hipertensão e dislipidemias & 5 & 12,8 \\
\hline Diabetes, hipertensão e outras doenças & 19 & 48,7 \\
\hline \multicolumn{3}{|c|}{$\operatorname{IMC}\left(\mathrm{kg} / \mathrm{m}^{2}\right)^{*}$} \\
\hline 15 a 25 & 15 & 38,4 \\
\hline 25,1 a 35 & 21 & 53,8 \\
\hline 35,1 a 45 & 3 & 7,6 \\
\hline
\end{tabular}

* calculado por meio da fórmula peso $(\mathrm{kg}) /$ altura $^{2}$ (metros) e classificado de acordo com as Diretrizes Brasileira de Obesidade em: normal (IMC $\leq 24,99 \mathrm{~kg} / \mathrm{m} 2$ ), sobrepeso (IMC $25-29,9 \mathrm{~kg} / \mathrm{m} 2$ ) e obeso $(\mathrm{IMC} \geq 30 \mathrm{~kg} / \mathrm{m} 2)$. 
Na Tabela 2 é possível observar que grande parte dos pacientes $(64,1 \%)$ recebeu diagnóstico de DM com idade entre 36 a 55 anos e pouco mais da metade $(51,28 \%)$ já possuía alguma complicação ocasionada pelo DM. É necessário destacar a já descrita dificuldade para determinar com exatidão o tempo da doença, devido ao período assintomático que precede o diagnóstico (1). Além disso, 69,23\% dos pacientes apresentava histórico familiar de DM com parentesco em primeiro grau, o que corrobora estudos anteriores que concluíram que familiares de primeiro grau de pessoas com DM 2 têm duas a seis vezes mais chances de desenvolver a doença, quando comparado com pessoas sem esse histórico familiar $(2,13)$.

Ainda, é possível observar que a maioria dos pacientes mantinha o acompanhamento médico com intervalo entre as consultas variando entre um a três meses $(71,8 \%)$ e realizava três ou mais testes glicêmicos diários de controle glicêmico. $\mathrm{O}$ intervalo entre as consultas pode dificultar a adequação do tratamento, pois os níveis de glicose sofrem muita variação até a determinação da concentração ideal da insulina, necessitando várias verificações da glicemia por dia para evitar casos de hipoglicemia ou hiperglicemia (2). Diante do exposto, é importante destacar o papel do farmacêutico no automonitoramento da glicose por esses pacientes. É esse profissional que pode auxiliar tanto na correta utilização do glicosímetro portátil quanto na interpretação do resultado promovendo assim a autonomia do paciente através do conhecimento acerca de sua terapia.

O Protocolo Clínico e Diretrizes Terapêuticas (PCDT) para DM tipo 2 publicado pelo Ministério da Saúde é um documento para orientar o tratamento, diagnóstico e acompanhamento de pacientes com a doença no sistema público de saúde (14). O PCDT para DM tipo 2 recomenda iniciar o tratamento somente com mudanças de hábitos de vida e reavaliação da resposta em 3 meses em pacientes com diagnóstico recente (inferior a 3 meses), pré-DM e baixo risco de eventos cardiovasculares. Entretanto, define que para a maioria dos pacientes é indicado o início terapêutico com metformina e mudanças de hábitos de vida após o diagnóstico (14).

Entre os medicamentos utilizados de maneira concomitante com a insulina, a metformina foi o antidiabético oral mais utilizado $(71,7 \%)$ por não apresentar risco de causar hipoglicemia, estabelecer benefícios em longo prazo de um controle intensivo da glicemia e exercer efeito favorável sobre o metabolismo lipídico, pois reduz os níveis de colesterol total, lipoproteínas de baixa densidade e triglicerídeos (15). A maioria dos pacientes não relatou dificuldade ou efeitos colaterais com a utilização dos hipoglicemiantes orais.

Tabela 2. Dados sobre o DM tipo 2 em pacientes insulinodependentes atendidos pela Farmácia Municipal Central de Santa Maria, RS, Brasil, no período de março a maio de 2019.

\begin{tabular}{|c|c|c|}
\hline Características & N & Valor $(\%)$ \\
\hline \multicolumn{3}{|c|}{ Diagnóstico (idade) } \\
\hline 23 a 35 & 10 & 25,6 \\
\hline 36 a 45 & 12 & 30,7 \\
\hline 46 a 55 & 13 & 33,3 \\
\hline 56 a 65 & 4 & 10,2 \\
\hline \multicolumn{3}{|c|}{ Histórico familiar de diabetes } \\
\hline Não & 7 & 17,9 \\
\hline \multirow{4}{*}{$\begin{array}{l}\text { Sim, familiares próximos } \\
\text { (mãe, pai, irmãos) }\end{array}$} & 13 & 33,3 \\
\hline & 12 & 30,7 \\
\hline & 2 & 5,1 \\
\hline & 5 & 12,8 \\
\hline \multicolumn{3}{|c|}{ Acompanhamento médico } \\
\hline 1 mês & 9 & 23 \\
\hline 2 meses & 10 & 25,6 \\
\hline 3 meses & 9 & 23 \\
\hline 4 meses & 1 & 2,5 \\
\hline 5 meses & 1 & 2,5 \\
\hline 6 meses & 9 & 23 \\
\hline \multicolumn{3}{|c|}{ Medicamentos hipoglicemiantes oral } \\
\hline Metformina & 28 & 71,7 \\
\hline Glibenclamida & 1 & 2,5 \\
\hline Metformina e glibenclamida & 4 & 10,1 \\
\hline Não & 6 & 15,3 \\
\hline \multicolumn{3}{|c|}{ Dificuldade de administrar o medicamento hipoglicemiante ora } \\
\hline Não & 37 & 94,8 \\
\hline Sim & 2 & 5,1 \\
\hline \multicolumn{3}{|c|}{ Efeito colateral o medicamento hipoglicemiante oral } \\
\hline Não & 33 & 84,6 \\
\hline Sim & 6 & 15,3 \\
\hline \multicolumn{3}{|c|}{ Testes de glicemia capilar diários } \\
\hline $1 x$ & 1 & 2,5 \\
\hline $2 x$ & 8 & 20,5 \\
\hline $3 x$ & 28 & 71,7 \\
\hline Mais de $3 x$ & 2 & 5,1 \\
\hline \multicolumn{3}{|c|}{ Acompanhamento com nutricionista } \\
\hline Não & 28 & 71,7 \\
\hline Sim & 11 & 28,2 \\
\hline
\end{tabular}


Interessantemente, somente $28,21 \%$ relatou acompanhamento com nutricionista (Tabela 2), indicando que esses pacientes podem estar consumindo alimentos que podem não ser adequados com sua condição clínica. Esse dado pode ser associado com os resultados demonstrados na Tabela 1, a qual indica que boa parte dos pacientes se encontra com sobrepeso e diferentes graus de obesidade.

Na Tabela 3 estão demonstrados dados sobre a utilização de insulina pelos pacientes participantes desse estudo. Pode ser observado que $69,22 \%$ dos pacientes iniciaram a administração da insulina há menos de oito anos, sendo a do tipo NPH (ação intermediária, 1-2 horas após administração) e Regular (ação rápida, 30-60 minutos após administração) as mais utilizadas. Essas insulinas eram fornecidas gratuitamente em algumas farmácias comerciais que eram credenciadas ao programa Farmácia Popular, cumprindo as diretrizes da Política Nacional de Assistência Farmacêutica (16). Cabe destacar que apesar das vantagens apresentadas pela insulina glargina, como maior previsibilidade no controle glicêmico e associação a menor risco de hipoglicemia noturna e ganho de peso (17), apenas $10,26 \%$ da população em estudo fazia o uso desse medicamento e esse fato pode estar associado a seu alto custo.

Foi observado ainda que, em grande parte dos casos, é o próprio usuário que administra a insulina, altera o local de aplicação e armazena de maneira adequada. Salienta-se que a maioria desses pacientes $(74,36 \%)$ obteve orientações por profissionais da saúde sobre a administração correta do medicamento, principalmente de médicos e enfermeiros. Apesar do farmacêutico ser o profissional do medicamento, a assistência farmacêutica ainda está em processo de implantação na saúde pública. As atribuições técnico-gerenciais do farmacêutico na saúde pública, como a racionalização, padronização e interação entre profissional e paciente foram regulamentadas somente em 2013 pela Resolução n 578 do Conselho Federal de Farmácia (18).

Os dados obtidos pelos diários e questionários de 18 pacientes que regressaram mensalmente à farmácia para o acompanhamento farmacoterapêutico durante os três meses de estudo, estão nas Tabelas 4 e 5, respectivamente. Na Tabela 4, observa-se que durante os dois primeiros meses
Tabela 3. Informações sobre a utilização de insulina por pacientes portadores de DM tipo 2 insulinodependentes atendidos pela Farmácia Municipal Central de Santa Maria, RS, Brasil, no período de março a maio de 2019.

\begin{tabular}{|c|c|c|}
\hline Caracteristicas & N & Valor $(\%)$ \\
\hline \multicolumn{3}{|c|}{ Início do tratamento com insulina } \\
\hline Mais de 10 anos & 6 & 15,3 \\
\hline De 10 a 8 anos & 6 & 15,3 \\
\hline Menos de 8 anos & 27 & 69,2 \\
\hline \multicolumn{3}{|c|}{ Tipo de insulina } \\
\hline NPH & 15 & 38,4 \\
\hline Regular e NPH & 21 & 53,8 \\
\hline Glargina e Aspart & 1 & 2,5 \\
\hline Glargina e Lispro & 1 & 2,5 \\
\hline Glargina e Glulisina & 1 & 2,5 \\
\hline \multicolumn{3}{|c|}{ Dificuldade na aplicação da insulina } \\
\hline Não & 35 & 89,7 \\
\hline Sim & 4 & 10,2 \\
\hline \multicolumn{3}{|c|}{ Aplicação da insulina } \\
\hline Próprio paciente & 37 & 94,8 \\
\hline Outro & 2 & 5,1 \\
\hline \multicolumn{3}{|c|}{ Local de aplicação } \\
\hline Alterna locais & 36 & 92,3 \\
\hline Não alterna o local & 3 & 7,6 \\
\hline \multicolumn{3}{|c|}{ Armazenamento da insulina } \\
\hline Tem conhecimento & 36 & 92,3 \\
\hline Não tem conhecimento & 3 & 7,6 \\
\hline \multicolumn{3}{|c|}{ Orientação sobre o uso de insulina } \\
\hline Não & 10 & 25,6 \\
\hline Médico & 17 & 43,5 \\
\hline Enfermeiro & 6 & 15,3 \\
\hline Farmacêutico & 2 & 5,1 \\
\hline Médico e enfermeiro & 4 & 10,2 \\
\hline
\end{tabular}

houve um aumento dos números de casos de hipoglicemia e hiperglicemia, bem como um aumento da média da glicemia mensal. No terceiro mês é possível observar uma redução nos parâmetros referidos acima, o que pode estar associado ao serviço de educação em saúde prestado pelos farmacêuticos durante o segundo mês, por meio das cartilhas sobre alimentação e atividade física que podem ter motivado os pacientes a mudarem seu estilo de vida, como demonstrado na Tabela 5. As informações adquiridas durante a intervenção farmacêutica realizada nesse trabalho demonstra- 
ram ser de grande importância, pois resultaram nas alterações positivas como novos hábitos alimentares e aumento na prática de exercício físico já que os pacientes relataram pouca ou nenhuma alteração em sua terapia farmacológica (Tabela
5). É necessário destacar que os gráficos gerados pelo glicosímetro portátil e as informações adquiridas foram enviados mensalmente ao médico responsável pelo tratamento dos pacientes incluídos nesse estudo.

Tabela 4. Dados dos diários distribuídos mensalmente aos pacientes portadores de DM tipo 2 insulinodependentes atendidos pela Farmácia Municipal Central de Santa Maria, RS, Brasil, no período de março a maio de 2019.

\begin{tabular}{|l|c|c|c|c|}
\hline \multicolumn{1}{|c|}{$1 \mathbf{x}$} & $\begin{array}{c}\mathbf{N}^{\circ} \text { de casos de } \\
\text { hipoglicemias }\end{array}$ & $\begin{array}{c}\mathbf{N}^{\circ} \text { de casos acima } \\
\text { de } 160 \mathrm{mg} / \mathrm{dL}\end{array}$ & $\begin{array}{c}\mathbf{N}^{\circ} \text { de casos acima } \\
\text { de } 300 \mathrm{mg} / \mathrm{dL}\end{array}$ & $\begin{array}{c}\text { Média da glicemia } \\
\mathrm{mensal}(\mathrm{mg} / \mathrm{dL})\end{array}$ \\
\hline $1^{\circ}$ Mês & 2,1 & 31,4 & 5,4 & 164,4 \\
\hline $2^{\circ}$ Mês & 2,1 & 32,8 & 5,7 & 173,6 \\
\hline $3^{\circ}$ Mês & 2 & 24,8 & 4,4 & 164,5 \\
\hline
\end{tabular}

Tabela 5. Dados dos questionários aplicados mensalmente aos pacientes portadores de DM tipo 2 insulinodependentes atendidos pela Farmácia Municipal Central de Santa Maria, RS, Brasil, no período de março a maio de 2019.

\begin{tabular}{|l|c|c|c|c|c|c|}
\hline & \multicolumn{2}{|c|}{$1^{\circ}$ MÊS } & \multicolumn{2}{|c|}{$2^{\circ}$ MÊS } & \multicolumn{2}{c|}{$3^{\circ}$ MÊS } \\
\cline { 2 - 6 } & Sim & Não & Sim & Não & Sim \\
\hline Alimentação Balanceada & $72,3 \%$ & $27,7 \%$ & $66,6 \%$ & $33,4 \%$ & $80 \%$ & $20 \%$ \\
\hline Doces & $27,7 \%$ & $72,3 \%$ & $44 \%$ & $56 \%$ & $20 \%$ & $80 \%$ \\
\hline Frutas & $77,7 \%$ & $22,3 \%$ & $72,3 \%$ & $27,7 \%$ & $80 \%$ & $20 \%$ \\
\hline Gorduras & $66,6 \%$ & 33,4 & $66,6 \%$ & $33,4 \%$ & $53,3 \%$ & $46,7 \%$ \\
\hline Atividade Física & $72,3 \%$ & $22,3 \%$ & $56 \%$ & $44 \%$ & $66,7 \%$ & $33,3 \%$ \\
\hline Alteração no hipoglicemiante oral & $11,1 \%$ & $88,8 \%$ & $13,3 \%$ & $86,6 \%$ & $0 \%$ & $100 \%$ \\
\hline Alteração no tipo de insulina & $16,6 \%$ & $83,3 \%$ & $0 \%$ & $100 \%$ & $0 \%$ & $100 \%$ \\
\hline Aplicação da insulina conforme prescrito & $94,4 \%$ & $5,5 \%$ & $93,3 \%$ & $6,6 \%$ & $100 \%$ & $0 \%$ \\
\hline
\end{tabular}

A Tabela 6 apresenta informações acerca dos medicamentos de outras classes utilizados pelos pacientes incluídos nesse estudo. Devido as características e as complicações associadas ao DM tipo 2, muitas vezes, se faz necessário o uso de uma grande variedade de medicamentos, sendo extremamente necessária a atuação clínica do farmacêutico, regulamentada pela Resolução $n^{\circ} 585 / 2013$ (19). Quando o DM tipo 2 não é controlado adequadamente com o tratamento farmacológico associado ao não farmacológico, essa doença pode evoluir para desenvolvimento de complicações crônicas, como a hipertensão arterial. Conforme observado em nosso estudo, a classe de medicamentos mais utilizadas pelos pacientes foi a dos anti-hipertensivos, incluindo losartana $(43,59 \%)$, enalapril $(28,21 \%)$ e hidroclorotiazida $(25,64 \%)$. Segundo a Diretriz Brasileira de Hipertensão Arterial cerca de 40\% dos pacientes diagnosticados com DM tipo 2 têm hipertensão, o que configura alto risco de desenvolver doença cardiovascular (20). Em segundo lugar estão os medicamentos utilizados para tratar dislipidemia, como a sinvastatina $(25,64 \%)$, que tem como objetivo a redução do risco cardiovascular (2). 
Tabela 6. Frequência de medicamentos de outras classes utilizados por pacientes DM tipo 2 insulinodependentes atendidos pela Farmácia Municipal Central de Santa Maria, RS, Brasil, no período de março a maio de 2019.

\begin{tabular}{|l|c|c|}
\hline \multicolumn{1}{|c|}{ Medicamentos } & $\begin{array}{c}\text { pacientes que } \\
\text { utilizavam (n=39) }\end{array}$ & $\begin{array}{c}\text { Frequência de uso } \\
(\%)\end{array}$ \\
\hline Losartana & 17 & 43,59 \\
\hline Enalapril & 11 & 28,21 \\
\hline Hidroclorotiazida & 10 & 25,64 \\
\hline Sinvastatina & 10 & 25,64 \\
\hline Omeprazol & 9 & 23,08 \\
\hline Ácido Acetilsalicilico & 8 & 20,51 \\
\hline Anlodipino & 7 & 17,95 \\
\hline Atenolol & 4 & 10,26 \\
\hline Fluoxetina & 4 & 10,26 \\
\hline Rosuvastatina & 4 & 10,26 \\
\hline Amitriptilina & 3 & 7,69 \\
\hline Espironolactona & 2 & 5,13 \\
\hline Metotrexato & 2 & 5,13 \\
\hline Prednisona & 1 & 5,13 \\
\hline Azatioprina & 2 & 2,56 \\
\hline Ciclosporina & 1 & 2,56 \\
\hline Clonidina & 1 & 2,56 \\
\hline Infliximabe & & 256 \\
\hline & 2 & \\
\hline
\end{tabular}

Com relação as IM, na Figura 1 é possível verificar que das $323 \mathrm{IM}$ encontradas, $6 \%$ foram classificadas como leves, $90 \%$ como moderadas e apenas $4 \%$ foram classificadas como graves. Somente as IM classificadas como graves serão aqui evidenciadas, uma vez que apresentam um maior risco à saúde podendo, muitas vezes, colocar a vida do paciente em risco (Tabela 7). Foi identificado um total de 11 IM graves, sendo a associação fluoxetina e amitriptilina a única que se repete.

Todos os entrevistados eram insulinodependentes, todavia cabe ressaltar não foram encontradas IM graves entre a insulina e os medicamentos em uso por estes pacientes. Os resultados aqui demonstrados auxiliam a reafirmar o importante papel do farmacêutico no âmbito do cuidado, uma vez que esse profissional pode interagir e colaborar com a equipe de saúde na tentativa de evitar ou identificar problemas relacionados a utilização dos medicamentos, promovendo assim o uso racional de medicamentos.

Medicamentos como a clonidina e atenolol são utilizados no tratamento da hipertensão arterial, condição essa relatada por mais de $76 \%$ dos pacientes desse estudo. Essa associação (Tabela 7) está relacionada ao aumento de bradicardia, hipotensão e, além disso, pode ocasionar em uma hipertensão rebote no paciente, quando ocorre a suspensão abrupta da clonidina (21).

Tabela 7. Interações medicamentosas classificadas como risco grave, entre pacientes DM tipo 2 insulinodependentes atendidos pela Farmácia Municipal Central de Santa Maria, RS, Brasil, no período de março a maio de 2019.

\begin{tabular}{|c|c|c|}
\hline Fármaco 1 & Färmaco 2 & Efeito \\
\hline Amitriptilina & Fluoxetina & Risco de toxicidade por ADT* (Sedação, boca seca, visão turva,) e $\uparrow$ de síndrome serotoninérgica. \\
\hline Atenolol & Clonidina & Efeitos farmacodinâmicos sinérgicos, como bradicardia e hipotensão. \\
\hline Enalapril & Espironolactona & $\uparrow$ do risco de hipercalemia. \\
\hline Espironolactona & Losartana & $\uparrow$ do risco de hipercalemia. \\
\hline Ciclosporina & Rosuvastatina & $\uparrow$ das concentrações plasmáticas de ciclosporina, podendo causar dor muscular, sensibilidade ou fraqueza. \\
\hline Metotrexato & Infliximabe & $\uparrow$ do risco de infecções. \\
\hline Azatioprina & Infliximabe & $\uparrow$ do risco de infecções. \\
\hline Prednisona & Infliximabe & $\uparrow$ do risco de infecções. \\
\hline Metotrexato & Omeprazol & $\uparrow$ dos níveis sanguíneos e os efeitos colaterais do metotrexato. \\
\hline Anlodipino & Sinvastatina & Risco de rabdomiólise. \\
\hline
\end{tabular}

*ADT: Antidepressivo tricíclico; $\uparrow:$ Aumento. 
Alguns fármacos utilizados rotineiramente em associação apresentaram IM grave como os medicamentos diuréticos poupadores de potássio (espironolactona) e os anti-hipertensivos inibidores de enzima conversa de angiotensina (IECA) (enalapril), bem como a espironolactona e bloqueadores dos receptores da angiotensina II (losartana). Esses estão relacionados ao aumento de potássio sérico e de arritmias cardíacas em pacientes portadores de DM, insuficiência renal, cardíaca e idade avançada (22).

Um outra IM grave encontrada entre medicamentos utilizados em associação para o tratamento de insuficiência cardíaca e hiperlipidemia foi entre os medicamentos anlodipino e a sinvastatina (Tabela 7). A coadministração desses medicamentos aumenta o risco de ocorrer miopatias e rabdomiólise, principalmente em pacientes portadores de DM e doenças cardiovasculares (23). Além disso, o anlodipino é responsável por aumentar as concentrações séricas de estatinas (24).

Ainda, foram encontradas IM graves para medicamentos utilizados para algumas doenças que não foram abordadas mais profundamente nesse estudo, como fluoxetina (inibidor seletivo da recaptação da serotonina) e a amitriptilina (antidepressivo tricíclico). A combinação desses pode resultar em toxicidade dos antidepressivos tricíclicos, mas tem sido utilizada para o tratamento de depressão resistente e outras doenças como dor crônica e enxaqueca (25). Também foi constatada a utilização de metotrexato e omeoprazol (Tabela 7). O uso concomitante desses medicamentos pode aumentar o risco de toxicidade do metotrexato, visto que essa associação é capaz de aumentar as concentrações séricas de metotrexato e também do seu metabólito ativo 7-hidroximetotrexato (26). A coadmistração de inibidores da bomba de prótons pode retardar a excreção do metotrexato e potencializar seus efeitos adversos (27).

Pouquíssimos pacientes relataram a utilização do medicamento infliximabe com metotrexato, infliximabe com azatioprina e infliximabe com prednisona. $\mathrm{O}$ infliximabe, um inibidor do Fator de Necrose Tumoral $\alpha$ (TNF $\alpha$ ), é utilizado no tratamento de diferentes doenças autoimunes (28). O uso do infliximabe associado com outros agentes imunossupressores (metotrexato, azatioprina e prednisona) aumenta o risco de desenvolvimento de infecções graves envolvendo múltiplos órgãos, sendo necessário a avaliação da relação risco versus benefício para o paciente (28).

Apenas um paciente relatou o uso concomitante de ciclosporina e rosuvastatina (Tabela 7). A ciclosporina é um fármaco imunossupressor utilizado na terapia de doenças autoimunes com o objetivo de prevenir e/ou tratar a rejeição de transplantes (24). Destaca-se que seu uso é limitado quando coadministrada com as estatinas, pois manifesta efeitos adversos importantes como aumento nas transaminases hepáticas e miopatia, além de elevar a concentração plasmática da rosuvastatina (29).

\section{CONCLUSÃO}

O DM é uma doença crônica que quando não tratada adequadamente leva ao desenvolvimento de complicações. A manutenção da glicemia e também da pressão arterial, condição demonstrada com bastante incidência neste trabalho, em níveis próximos ao desejável pode auxiliar a retardar ou até mesmo prevenir as complicações do DM. $\mathrm{O}$ estudo reafirma que a atuação do farmacêutico por meio de serviços como o acompanhamento farmacoterapêutico e de educação em saúde é fundamental na promoção do autocuidado e auxilia sobremaneira no controle de doenças crônicas como o DM. Em decorrência do caráter crônico desta doença, a demanda de medicamentos hipoglicemiantes, anti-hipertensivos, antilipêmicos, além de outros se faz necessário. Situação que pode levar os pacientes a um quadro de polifarmácia, aumentando o índice de ocorrência de interações medicamentosas.

Ademais, é de suma importância que o farmacêutico atue juntamente com os outros profissionais da saúde na elaboração estratégias com planos de intervenções especializados para essa parcela da população, uma vez que este profissional garante um atendimento complementar e de qualidade, sendo capaz de orientar e intervir no tratamento de um paciente de maneira a reduzir potenciais riscos através do uso racional e seguro de seus medicamentos. 
1. FID. Atlas de Diabetes $9^{\circ}$ ed. Federação Internacional de Diabetes, 2019, p. 176.

2. SBD. Diretrizes da Sociedade Brasileira de Diabetes, 2019-2020. Sociedade Brasileira de Diabetes. Disponível em: <https://www.diabetes.org.br/profissionais/images/ DIRETRIZES-COMPLETA-2019-2020.pdf >

3. Schmidt MI, Hoffman JF, Diniz MFS, Lotufo PA, Griep RH, Bensenor IM, Mill JG, Barreto SM, Aquino EML, Duncan BB. High prevalence of diabetes and intermediate hyperglycemia - The Brazilian Longitudinal Study of Adult Health (ELSA-Brasil). Diabetol Metab Syndr. 2014;6:123. DOI: $10.1186 / 1758-5996-6-123$.

4. Eik Filho WE, Bonjorno LP, Franco AJM, Santos MLA, Souza EM, Marcon SS. Evaluation, intervention, and follow-up of patients with diabetes in a primary health care setting in Brazil: the importance of a specialized mobile consultancy. Diabetol Metab Syndr. 2016;8(56). DOI: 10.1186/s13098-016-0173-1.

5. BRASIL. Lei Federal $n^{\circ} 11.347$, de 27 de setembro de 2006. Dispõe sobre a distribuição gratuita de medicamentos e materiais necessários à sua aplicação e à monitoração da glicemia capilar aos portadores de diabetes inscritos em programas de educação para diabéticos. Disponível em: https://www.planalto.gov.br/ccivil_03/_ato20042006/2006/lei/111347.htm.

6. BRASIL. Ministério da Saúde. Portaria $\mathrm{n}^{\circ} 2.583$, de 10 de outubro de 2007. Define elenco de medicamentos e insumos disponibilizados pelo Sistema Único de Saúde, nos termos da Lei ${ }^{\circ} 11.347$, de 2006, aos usuários portadores de diabetes mellitus. Disponível em: https://bvsms.saude.gov.br/bvs/saudelegis/gm/2007/ prt2583_10_10_2007.html.

7. Souza SPS., Lima RAG. Condição crônica e normalidade: rumo ao movimento que amplia a potência de ser feliz. Rev Lat Am Enfermagem. 2007;15(1): 156-164.

8. MINAS GERAIS. Prefeitura Municipal. Secretaria Municipal de Saúde de Belo Horizonte. Protocolo de Diabetes Mellitus. Disponível em: http://goo.gl/6UgvJ.

9. Zavatini MA, Obreli-Neto PR, Cuman RKN. Estratégia saúde da família no tratamento de doenças crônicodegenerativas: avanços e desafios. Rev Gaúcha Enferm. 2010;31(4). DOI: 10.1590/S1983-14472010000400006.

10. Salin AB, Bandeira MSN, Freitas PRNO, Serpa I. Diabetes Mellitus tipo 2: perfil populacional e fatores associados à adesão terapêutica em Unidades Básicas de Saúde em Porto Velho-RO. Rev. Eletrônica Acervo Saúde. 2019;33:1-9. DOI: 10.25248/reas.e1257.2019.

11. Duncan BB, Schmidt MI, Cousin E, Ishitani LH, Malta DC, Naghavi M, Mooney M, Schmidt MI. The burden of diabetes and hyperglycemia in Brasil - past and present: findings from the Global Burden of Disease Study 2015. Rev Bras Epidemiol. 2017;20(1). DOI: 10.1590/19805497201700050008.

12. AAD. Standands of medical care in diabetes. American Association Diabetes Disponível em:< https://care. diabetesjournals.org/>.

13. Rosenbloom AL, Joe JR, Young RS, Winter WE. Emerging epidemic of type 2 diabetes in youth. Diabetes Care. 1999; 22(2):345-354.

14. BRASIL. Ministério da Saúde/Secretaria de Ciência, Tecnologia, Inovação e Insumos Estratégicos em Saúde. Portaria SCTIE/MS n ${ }^{\circ} 54$, de 11 de novembro de 2020. Aprova o Protocolo Clínico e Diretrizes Terapêuticas do Diabete Melito Tipo 2. https://www. in.gov.br/en/web/dou/-/portaria-sctie/ms-n-54-de-11-denovembro-de-2020-288070624.

15. Rojas, LB. Metformin: an old but still the best treatment for type 2 diabetes. Diabetol Metab Syndr. 2013;5(1):6. DOI: 10.1186/1758-5996-5-6.

16 BRASIL. Ministério da Saúde. Conselho Nacional de Saúde. Resolução no 338, de 06 de maio de 2004. aprova a Política Nacional deAssistência Farmacêutica, estabelecida em alguns princípios: a Política Nacional de Assistência Farmacêutica, a Assistência Farmacêutica e ações referentes a esta.

17. Hirsch IB. Insulin analogues. N Engl J Med. 2005;352(2):174-183. DOI: 10.1056/NEJMra040832.

18. CFF. Resolução $n^{0} 578$ de 26/07/2013. Regulamenta as atribuições técnicogerenciais do farmacêutico na gestão da assistência farmacêutica no âmbito do Sistema Único de Saúde (SUS). Conselho Federal de Farmácia. Diário Oficial da União de 19/08/2013. Disponível em: https:// www.cff.org.br/userfiles/file/resolucoes/578.pdf.

19. CFF. Resolução no 585 de 29/08/2013. Regulamenta as atribuições clínicas do farmacêutico e dá outras providências. Conselho Federal de Farmácia. Diário Oficial da União de 25/09/2013. Pág. 159. Disponível em: https://www.cff.org.br/userfiles/file/resolucoes/585.pdf.

20. Malachias MVB, Souza WKSB, Plavnik FL, Rodrigues CIS, Brandão AA, Neves MFT, Bortolotto LA, Franco RJS, Poli-de-Figueiredo CE, Jardim PCBV, Amodeo C, Barbosa ECD, Koch V, Gomes MAM, Paula RB, Póvoa RMS, Colombo FC, Ferreira Filho S, Miranda RD, Machado CA, Nobre F, Nogueira AR, Mion Júnior D, Kaiser S, Forjaz CLM, Almeida FA, Martim JFV, Sass N, Drager LF, Muxfeldt E, Bodanese LC, Feitosa AD, Malta D, Fuchs S, Magalhães ME, Oigman W, Moreira Filho O, Pierin AMG, Feitosa GS, Bortolotto MRFL, Magalhães LBNC, Silva ACS, Ribeiro JM, Borelli FAO, Gus M, Passarelli Júnior O, Toledo JY, Salles GF, Martins LC, Jardim TSV, Guimarães ICB, Antonello IC, Lima Júnior E, Matsudo V, Silva GV, 
Costa LS, Alessi A, Scala LCN, Coelho EB, Souza D, Lopes HF, Gowdak MMG, Cordeiro Júnior AC, Torloni MR, Klein MRST, Nogueira PK, Lotaif LAD, Rosito GBA, Moreno Júnior H. $7^{\text {a }}$ Diretriz Brasileira de Hipertensão Arterial. Arq Bras Cardiol. 2016;107(3):1-104. DOI: 10.5935/ abc.20160151.

21. Secoli, SR, Danzi NJ, Lima FFF, Lorenzi Filho G, Cesar LAM. Interações Medicamentosas em Pacientes Coronariopatas. Rev Bras Cardiol. 2012; 25(1):11-18.

22. Mibielli P, Rozenfeld S, Matos GC, Acurcio FA. Interações medicamentosas potenciais entre idosos em uso dos anti-hipertensivos da Relação Nacional de Medicamentos Essenciais do Ministério da Saúde do Brasil. Cad. Saúde Pública. 2014; 30(9):1947-19566. DOI: 10.1590/0102311 X00126213.

23. Nguyen KA, Li L, Lu D, Yazdanparast A, Wang L, Kreutz RP, Whipple EC, Schleyer TK. A comprehensive review and meta-analysis of risk factors for statin-induced myopathy. Eur J Clin Pharmacol. 2018;74(9):1099-1109. DOI: 10.1007/ s00228-018-2482-9.

24. Rang, HP, Ritter, JM, Flower RJ, Henderson G. Rang \& Dale Farmacologia. 8 ed., Elsevier, 2016.
25. Brunton LL, Lazo JS, Parker KL. As Bases Farmacológicas da Terapêutica de Goodman \& Gilman. 11 ed., Mc-Graw-Hill, 2010.

26. Reid T, Yuen A, Catolico M, Carlson RW. Impacto of omeprazole on the plasma clearance of methotrexate. Cancer Chemother Pharmacol. 1993;33(1):82-84. DOI: 10.1007/ BF00686028.

27. Suzuki KD, Doki K, Homma M, Tamaki H, Hori S, Ohtani H, Sawada Y, Kohda Y. Co-administration of proton pump inhibitors delays elimination of plasma methotrexate in high-dose methotrexate therapy. Br J Clin Pharmacol. 2009;67(1):44-49. DOI: 10.1111/j.13652125.2008.03303.x.

28. Andersen NN, Jess T. Risk of infections associated with biological treatment in inflammatory bowel disease. World J Gastroenterol. 2014;20(43):16014-16019. DOI: 10.3748/ wjg.v20.i43.16014.

29. Izar MCO. Tratamento hipolipemiante em situações especiais: pós-transplante e/ou terapia imunossupressora. Arq Bras Cardiol. 2005;85(5):50-57. DOI: 10.1590/ S0066-782X2005002400013 